Pneumologe 2013 $\cdot$ 10:391-393

DOI 10.1007/s10405-013-0704-5

Online publiziert: 1. November 2013

(c) Springer-Verlag Berlin Heidelberg 2013

\title{
C. Taube
}

Department of Pulmonology, Leiden University Medical Center, Leiden

\section{Asthma}

klinischer Entwicklung, als Beispiel seien monoklonale Antikörper gegen verschiedene Entzündungsmediatoren wie Interleukin-(IL-)4, IL-5 oder IL-13 genannt. Entsprechende Patientengruppen zu erkennen, die auf solche Therapien ansprechen könnten, wird dabei eine große Aufgabe in der Asthmadiagnostik der $\mathrm{Zu}$ kunft sein. Diese Entwicklungen sind in diesem Heft in dem Beitrag „Aktuelles in der Asthmadiagnostik“ zusammengefasst.

\section{》) Geringere Compliance bei schwangeren Asthmapatientinnen}

Ein Phänomen, das bei Patienten mit Asthma auftreten kann, ist die sog. belastungsindizierte Bronchokonstriktion. Gerade bei Leistungssportlern treten diese Beschwerden gehäuft auf, unterscheiden sich aber in verschiedenen Aspekten von einem klassischen Asthma. Wichtige klinische Unterschiede und Behandlungsmöglichkeiten sind in dem Beitrag „Athleten, Asthma und Anstrengung " beschrieben.

Eine weitere Patientengruppe, bei der häufig Fragen bei der Behandlung auftreten, sind Schwangere mit Asthma. Bei ca. einem Drittel der Frauen wird dabei das Asthma besser, bei einem Drittel ist es gleichbleibend und bei einem Drittel nehmen die Beschwerden erheblich zu. Gerade in dieser Patientengruppe ist aber mit einer geringeren Compliance in Bezug auf den Einsatz der Medikation aufgrund von Ängsten vor eventuellen Nebenwirkungen zu rechnen. In dem Beitrag „Asthma und Schwangerschaft" finden Sie aktuelle Empfehlungen für die Therapie von schwangeren Patientinnen mit Asthma.

Unter dem Überbegriff schweres Asthma versammeln sich in der klinischen Praxis hoch komplexe Patienten. Dabei ist immer die Überprüfung der
Diagnose und Ausschluss von Differentialdiagnosen notwendig. Ein fließender Übergang wird insbesondere zum Churg-Strauss-Syndrom (CSS) beobachtet. Die wesentlichen Unterschiede zwischen Asthma und CSS werden in dem Beitrag „Asthma bronchiale und ChurgStrauss-Syndrom“ dargestellt.

Als Behandlungsoption für Patienten mit schwerem Asthma ist inzwischen auch die bronchiale Thermoplastie in Europa zugelassen. Es handelt sich dabei um ein interventionelles Verfahren, welches inzwischen in mehreren, teilweise randomisierten und kontrollieren Studien untersucht ist. In dem Artikel „Bronchiale Thermoplastie: Interventionelle Therapie bei Asthma bronchiale" werden diese Studienergebnisse zusammengefasst und kritisch bewertet.

Sowohl die Diagnostik als auch die Therapie für das Syndrom Asthma entwickeln sich weiter und wir denken, Ihnen in diesem Heft eine aktuelle Übersicht über wichtige Themen wie Diagnostik, Differentialdiagnostik und Therapie zu geben. Wir haben dieses Heft bewusst auf bereits zugelassene Behandlungsoptionen beschränkt. Weitere therapeutische Optionen und eine neue deutsche Asthmaleitlinie sind aber bereits am Horizont erkennbar.

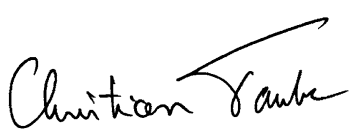

Prof. Dr. C. Taube

\section{Korrespondenzadresse}

Prof. Dr. C. Taube

Department of Pulmonology, Leiden University Medical Center

Albinusdreef 2, 2333 ZA Leiden

The Netherlands

C.Taube@lumc.nl 


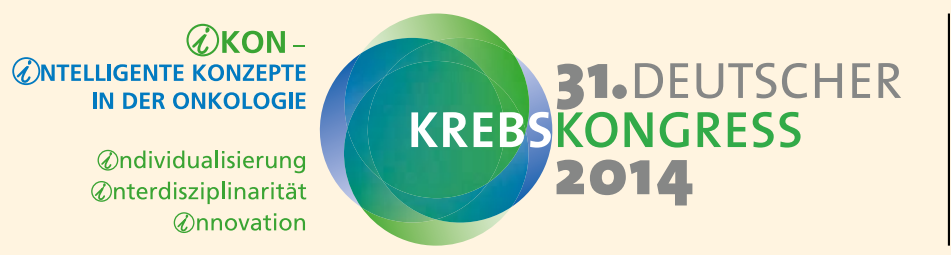

MESSE BERLIN - SÜDGELÄNDE 19. - 22. FEBRUAR 2014

www.dkk2014.de

\section{Innovativ, kompakt, direkt, interdisziplinär: Deutscher Krebskongress 2014}

Die Vorbereitungen laufen auf Hochtouren: Vom 19. bis 22. Februar 2014 findet in Berlin der 31. Deutsche Krebskongress (DKK) statt. An vier Tagen informieren sich auf diesem größten und wichtigsten deutschsprachigen Kongress zur Krebsdiagnostik und Krebstherapie rund 10.000 Experten über die jüngsten Entwicklungen und diskutieren die Aufgaben von heute und morgen - kompakt, intensiv, interdisziplinär und im direkten Gespräch von Mensch zu Mensch. Kongresspräsident 2014 ist Prof. Dr. Michael Hallek, Köln. Erstmals richten die Deutsche Krebsgesellschaft und die Deutsche Krebshilfe den Kongress gemeinsam aus. Die gebündelte Kompetenz der beiden Organisationen ermöglicht es, den DKK thematisch und organisatorisch weiterzuentwickeln.

Worum es geht: Innovationen, Interdisziplinarität, Individualisierung Drei wesentliche Herausforderungen, mit denen sich die Krebsmedizin derzeit beschäftigt, sind bei der Programmplanung im Mittelpunkt: verstärkte Förderung von Interdisziplinarität, zügige Integration und Finanzierung von Innovationen und zunehmende Individualisierung von Therapieentscheidungen nach einer molekular-genetischen Diagnostik. All das erfordert "Intelligente Konzepte in der Onkologie (iKon)" und definiert das Motto des DKK 2014. Zum einen bietet das Programm Aktuelles zur Diagnostik und Therapie der Haupttumorarten und der seltenen Tumoren. Ein Thema, das dabei ebenfalls diskutiert werden soll, betrifft die Forschung: Wie kann man künftig in Deutschland eine starke und innovative Krebsforschung finanziell und organisatorisch sichern? Zum anderen widmet sich der Kongress dem großen Thema der optimalen Versorgungsqualität durch Leitlinien, Zertifizierungen und Krebsregister. Wie entwickelt man die Qualitätssicherung weiter und gestaltet zugleich die Dokumentation effizient und effektiv? Der Weg dorthin soll auf dem Kongress diskutiert werden. Neben dem wissenschaftlichen Programm befasst sich der DKK mit gesundheitspolitischen Themen. Knapp fünf Monate nach der Bundestagswahl stehen die gesundheitspolitischen Ziele aus dem neuen Koalitionsvertrag zur Diskussion. Eines davon ist der Nationale Krebsplan. In vielen Handlungsfeldern wurden bereits Ergebnisse erzielt, viele Fragen sind aber noch offen, beispielsweise in der Psychoonkologie: Versorgungsangebote existieren - aber wie kommt man zu bedarfsgerechten, einheitlichen Versorgungsstandards und einer nachhaltigen Finanzierung?

\section{Interdisziplinär auch im Format:} fachübergreifende Sitzungen

Diese und andere politische und wissenschaftliche Themen sollen auf dem Krebskongress diskutiert werden. Besonderer Wert liegt auf der interdisziplinären Zusammensetzung aller Diskussionsformate: Operateure, Strahlentherapeuten und Internisten sowie Psychoonkologen, Palliativmediziner und alle anderen, die an der Versorgung des Patienten beteiligt sind, werden auf dem DKK 2014 nicht in getrennten Sitzungen diskutieren, sondern gemeinsam.

\section{Im besonderen Fokus: der klinische Nachwuchs}

Dass der DKK 2014 erneut auf großes Interesse stößt, zeigt die Zahl der eingereichten Abstracts: 443. In der Phase der Beitragseinreichung wurde explizit der klinische Nachwuchs aufgefordert, sich mit seinen Arbeitsergebnissen vorzustellen. Der Kongress unterstützt dessen Begeisterung für die Onkologie: durch eine besondere Würdigung von guten Abstracts, durch vielfältige Möglichkeiten zur Präsentation und zu freien Vorträgen oder durch eine innovativ gestaltete Postersitzung.

\section{Im persönlichen Gespräch: Betroffene und Experten}

Vier Tage Kongress - das sind vier Tage Kommunikation über Krebs aus verschiedenen medizinischen und gesundheitspolitischen Perspektiven. Ziel aller Fachdiskurse ist immer das Patientenwohl. Aber auch für das persönliche Gespräch zwischen Patienten und Experten bietet der DKK Zeit und Raum: Am Samstag, dem 22. Februar, sind Betroffene, Angehörige und Interessenten zum 6. Krebsaktionstag, einem Informationstag für die Öffentlichkeit, eingeladen. Dann stehen Mediziner, Fachgesellschaften, Vertreter der Pflege, Selbsthilfegruppen und Patientenorganisationen für Gespräche bereit.

Alle Informationen, das Vorprogramm und die Onlineanmeldung gibt es auf der offiziellen Webseite www.dkk2014.de.

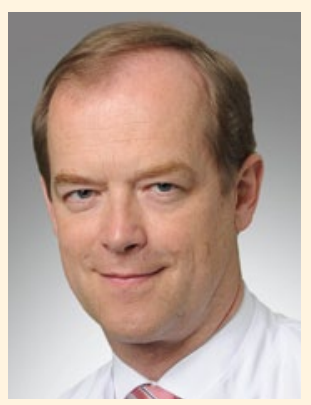

„Wer sich in kürzester Zeit auf den neuesten wissenschaftlichen und gesundheitspolitischen Stand bringen möchte, der muss zum DKK 2014 kommen. Aber noch wichtiger als Information ist aus meiner Sicht der Diskurs: Lassen Sie uns die Gelegenheit nutzen, die sich an vier Tagen bietet, um über die brennenden Fragen in der Onkologie zu diskutieren. Ich lade Sie herzlich dazu ein."

Prof. Dr. Michael Hallek, Präsident des Deutschen Krebskongresses 2014 


\section{Hier steht eine Anzeige.}

Springer 